\title{
H1-5 Gene
}

National Cancer Institute

\section{Source}

National Cancer Institute. H1-5 Gene. NCI Thesaurus. Code C162849.

This gene is involved in nucleosome organization. 